\title{
Active control of laminar-turbulent transition
}

\author{
By H. W. LIEPMANN AND D. M. NOSENCHUCK \\ California Institute of Technology, Pasadena, CA 91109, U.S.A.
}

(Received 21 November 1981)

Instability waves, commonly called T-S waves, can be introduced in a laminar boundary layer by periodic heating of flush-mounted heating elements. Experiments have demonstrated that nearly complete cancellation of a T-S wave excited in this way can be achieved by using a second downstream heating element with a suitable phase shift. As one application of the technique, a single element together with a feedback loop activated by measured wall shear stress has been used to reduce the amplitude of naturally occurring laminar instability waves. A significant increase in the transition Reynolds number has been achieved.

In the preceding paper (Liepmann, Brown \& Nosenchuck 1982, hereinafter referred to as I), we have demonstrated the use of a new technique to excite laminar instability waves as a means for the study and possible control of boundary-layer transition.

The transition to turbulent motion proceeds in two steps: there is first a comparativeIy slow amplification of the essentially two-dimensional T-S waves, which is then followed by a rapid three-dimensional dynamic instability. Using the language of chemical kinetics, the first process is rate-controlling. We have succeeded in using active feedback control to reduce the wave amplitude and hence to delay transition.

The experiments were carried out with the apparatus and in the facility described in $\mathrm{I}$. The distribution of the activating and shear-stress-measuring elements are shown in figure 1. The quadratic dependence of ohmic heating on the heating current usually leads to a doubling of the temperature-fluctuation frequency compared to the driving-current frequency. This was the case in the experiments reported in $\mathrm{I}$. The present set of experiments uses a digital function generator which permits a square-root-type voltage to be applied so that the temperature and current frequencies are the same. This modification is very helpful for the design of the feedback loop.

Figure 2 demonstrates the use of this technique in cancelling or reinforcing a deliberately excited T-S wave. A heating element, H0 (at $x=2$ in., corresponding to $\left.R_{x}=65000\right)$, excites the wave. A second element, H1 ( $x=2 \cdot 75$ in., $\left.R_{x}=90000\right)$, can either reinforce or cancel the wave, depending on the relative phases of the driving voltages. Figure 2 shows the wall stress fluctuations measured at $x=8 \mathrm{in} ., R_{x}=250000$, $2(a)$ with zero input, $2(b)$ with input from $\mathrm{HO}$ only, $2(c)$ with reinforcement, and $2(d)$ with cancellation, using an input from $\mathrm{H} 1$ phased appropriately. Figures $2(e, f)$ show the corresponding input voltages to the two heating elements. The almost-complete cancellation of the excited T-S wave is evident from a comparison of $2(b)$ with $2(d)$. 


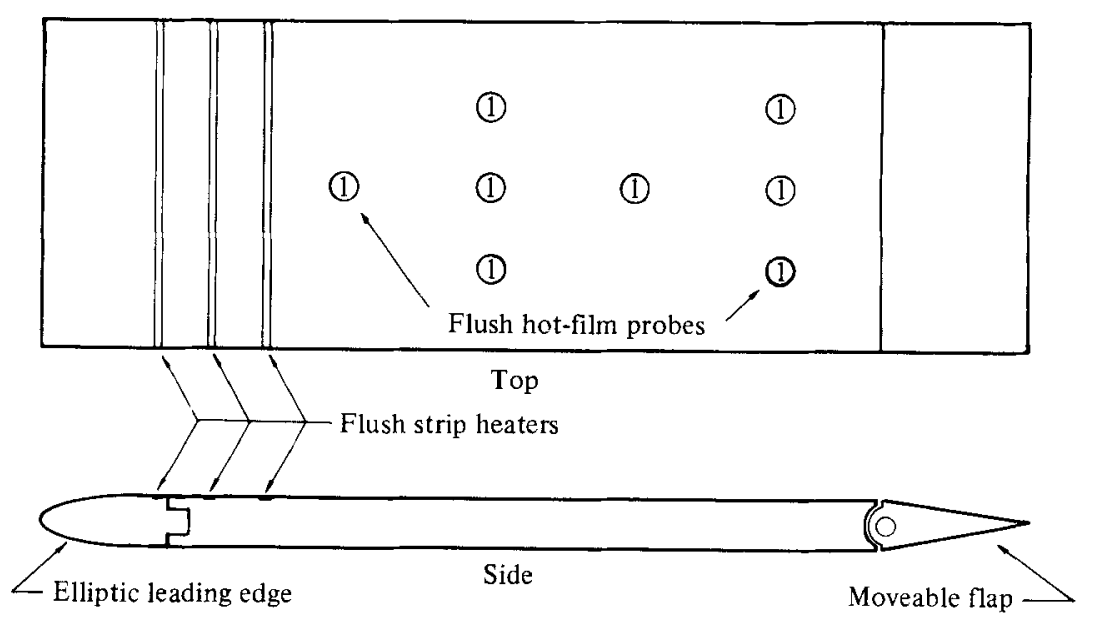

FIGURE 1. Flat-plate layout.
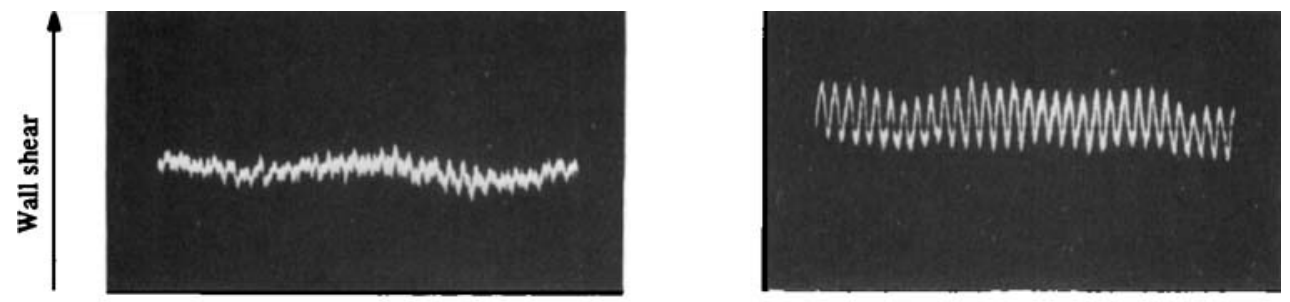

(a) No forcing

Time (100 ms/div)

(b) Response to upstream heater
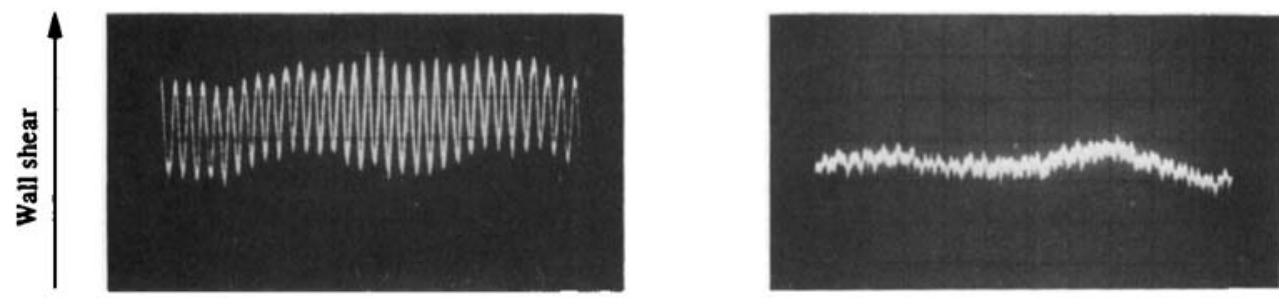

(c) Reinforcement Time (100 ms/div)

(d) Cancellation
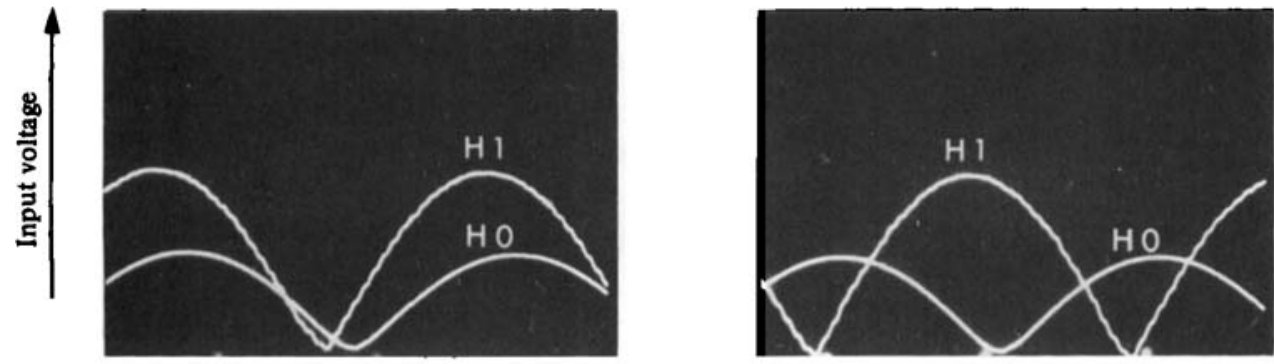

(e) Input signals for reinforcement $\stackrel{\text { Time }(5 \mathrm{~ms} / \mathrm{div})}{\longrightarrow}(f)$ Input signals for cancellation FIGURE 2. Forced wave interactions at $R_{x}=2.5 \times 10^{5}$. 


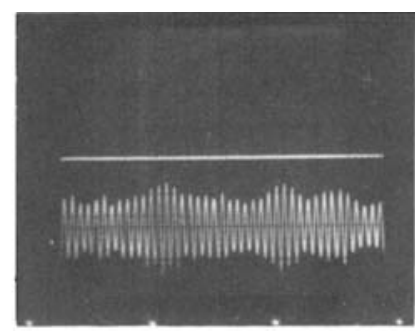

(a) Feedback off

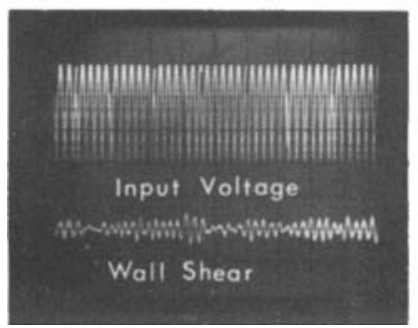

(b) Feedback on: out-of-phase cancellation

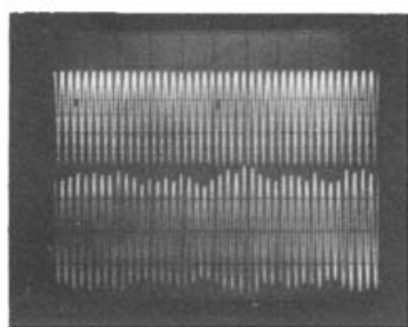

(c) Feedback on : in-phase reinforcement

FiguRe 3. Feedback control of naturally occurring T-S waves at $R_{x}=4 \cdot 6 \times 10^{5}$. All photos, $100 \mathrm{~ms} /$ div.

Note that the phase of the T-S wave at the location of $\mathrm{H} 1$ differs from the phase at $\mathrm{H} 0$ by $\Delta x / c$, where $\Delta x$ is the distance between $\mathrm{H} 0$ and $\mathrm{H} 1$, and $c$ is the wave velocity. The corresponding voltages, of course, have to reflect this fact. In figure 2 , it just happens that $\Delta x$ is nearly equal to $\lambda$, the wavelength of the T-S wave.

A few experiments were carried out with heating element $\mathrm{H}_{2}$ at $x=4 \mathrm{in}$, at $R_{x}=130000$. The results were the same, but of course $\Delta x \neq \lambda$.

Figure 3 shows the control of a 'natural' T-S wave using a feedback loop. The upper trace in each of the three parts of figure 3 is the input voltage to the single heating element. The lower trace is the measured shear-stress fluctuation downstream. Figure $3(a)$ shows the 'natural' shear-stress fluctuation; figure $3(b)$ shows cancellation; and figure $3(c)$ shows reinforcement. The reduction in the amplitude of the shear-stress fluctuation by this cancellation technique is evident. This reduction in wave amplitude results in a marked delay in the transition to turbulence, as shown in the dye-streak flow visualization of figures $4(a, b)$. Similarly, transition is accelerated if the actuating strip is driven in the opposite phase relationship, as seen in figure $4(c)$. The corresponding transition Reynolds numbers are about $8 \times 10^{5}, 1 \cdot 1 \times 10^{6}$, and $5 \times 10^{5}$, respectively.

These experiments demonstrate the possibility of using active control to delay (or accelerate) the transition to turbulence. This approach differs in an essential way from previous attempts to delay transition, which were based on modification of the mean-velocity profile. In our approach, the developing perturbations are cancelled, or at least reduced in amplitude, by using phase control. This increase in transition Reynolds number was achieved with an expenditure of $10 \mathrm{~W}$ of electrical power. This number should be compared with the $2000 \mathrm{~W}$ required to achieve the same increase under identical flow conditions using steady heating of the whole plate. We believe that these experiments are the first to use active control in any turbulencerelated problem. 
(a)

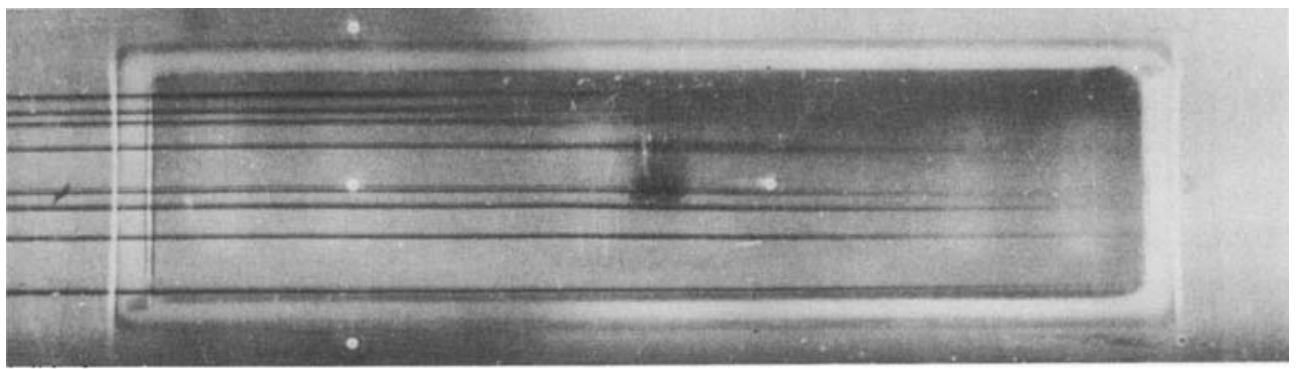

(b)

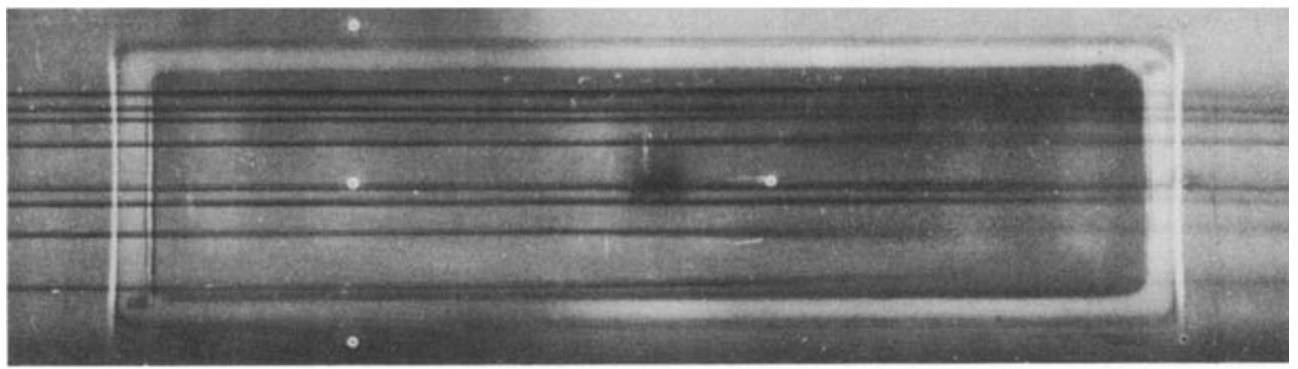

(c)

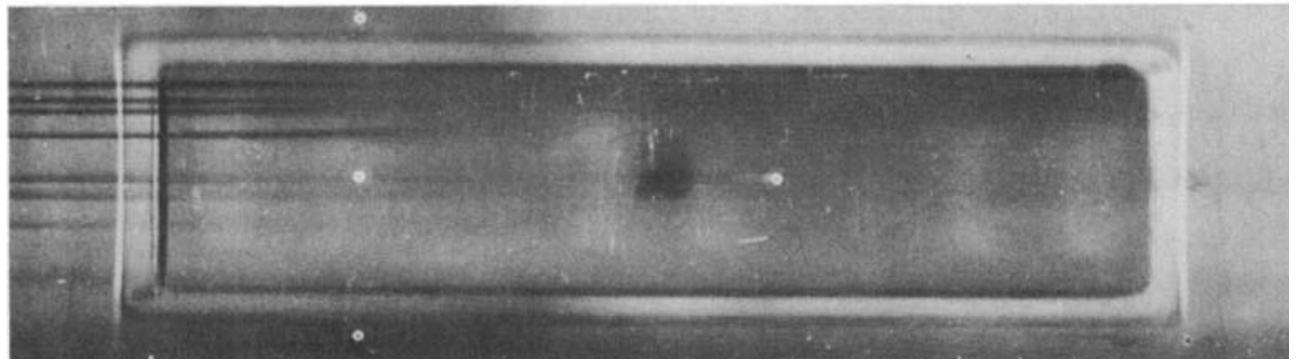

Frgure 4. Dye streaks in the boundary layer. Flow is left to right. (a) Natural transition, (b) cancellation of a natural wave, (c) reinforcement of a natural wave.

The competent assistance of Steve Taylor, Harry F. Robey, and Jack Kingan was essential for the success of this experiment. The authors gratefully acknowledge the support of this research by the Office of Naval Research under ONR contract N0001481-K-0551.

\section{REFERENCE}

Liepmann, H. W., Brown, G. L. \& Nosenchuck, D. M. 1982 Control of laminar instability waves using a new technique. $J$. Fluid Mech. 118, 187-200. 